\title{
METABOLITOS NITROGENADOS EN EL HÁMSTER DORADO ALIMENTADO A BASE DE HARINA DE LOMBRIZ (EISENIA SPP.)
}

\author{
NITROGENED METABOLITES IN GOLDEN HAMSTER FED WITH PROTEIC DIETS \\ BASED ON EISENIA SPP. MEAL
}

\author{
García, D.E. ${ }^{1 *}$, Cova, L.J. ${ }^{2}$, Briceño, S. ${ }^{3}$, Scorza D., J.V. ${ }^{2}$, Montilla, F. ${ }^{2}$, Medina, M.G. ${ }^{1}$, \\ Perea, F. ${ }^{1}$ y González, D. ${ }^{1}$
}

'Departamento de Ciencias Agrarias. Núcleo Universitario Rafael Rangel. Universidad de Los Andes (ULA). Estado Trujillo. Venezuela. *dagamar8@hotmail.com ${ }^{2}$ Instituto Experimental José Witremundo Torrealba. ULA. Estado Trujillo. Venezuela. ${ }^{3}$ Laboratorio de Bioanálisis. Instituto Venezolano del Seguro Social. Trujillo. Estado Trujillo. Venezuela.

\section{PalabRAS ClaVe ADICIONALES}

Proteínas séricas. Transaminasa. Ácido úrico. Urea sérica. Creatinina. Química sanguínea.

\section{RESUMEN}

Se evaluó en el plasma sanguíneo del hámster dorado (Mesocrisetus auratus L.) el contenido total de proteína (PT), albúmina (Alb), globulinas $(\mathrm{Glb})$, urea $(\mathrm{U})$, ácido úrico $(\mathrm{AU})$, creatinina $(\mathrm{Ct})$ y los niveles de transaminasas (glutámico oxalacética (TGO) y glutámico-pirúvica (TGP)) a partir del consumo de dietas (proteína bruta; $\mathrm{PB}=13,5$ $19,6 \%$ ) con harina de lombriz roja (HL) (Eisenia spp.) y dos alimentos convencionales (Conejarina $\AA ; \mathrm{PB}=11,4 \%$ y Ratarina $\AA ; \mathrm{PB}=21,1 \%$ ). $\mathrm{La}$ concentración de PT, Alb, Glb y Ct osciló entre 5$6 ; 2-3 ; 2-3 \mathrm{~g} / \mathrm{dL}$ y $0,3-0,4 \mathrm{mg} / \mathrm{dL}$, respectivamente, sin observarse diferencias significativas entre las dietas $(p>0,05)$. En todo el experimento los niveles de $U$ y AU fluctuaron entre 23-101 y 3-9 $\mathrm{mg} / \mathrm{dL}$, respectivamente, en función del nivel de $\mathrm{PB}$, la inclusión de $\mathrm{HL}$ y el momento de muestreo. Los niveles de transaminasas fueron elevados y variables, acorde al valor proteico de las dietas (TGO: 101-699 y TGP: 54-890 UI/L). Se comprobó que los contenidos séricos de PT, Glb, Alb y $\mathrm{Ct}$ fueron independientes de la inclusión de HL y los niveles de PB de cada alimento; mientras que la concentración de $U\left(R^{2}=0,509^{*}\right)$, $\operatorname{TGO}\left(R^{2}=0,661^{* *}\right)$ y TGP $\left(R^{2}=0,524^{*}\right)$ se encontraron relacionados con los valores proteicos de las raciones, y el contenido de $U\left(R^{2}=0,502^{*}\right)$ y $A U\left(R^{2}=0,531^{*}\right)$ con la inclusión de HL. La adición de HL en proporciones de 24,27 y $30 \%$ a dietas formuladas para $M$.

Recibido: 22-11-10. Aceptado: 8-6-11.

\section{Additional KeYWORDS}

Seric proteins. Transaminase. Uric acid. Seric urea. Creatinine. Blood chemistry.

auratus aumenta significativamente los valores normales de $\mathrm{U}, \mathrm{AU}$ y transaminasas.

\section{SUMMARY}

Content of total protein (PT), albumin (Alb), globulins (GIb), urea (U), uric acid (AU), creatinine $(\mathrm{Ct})$ and transaminase levels (oxalacetic-glutamic (TGO) and piruvic-glutamic (TGP)) was evaluated in the blood plasm of golden hamster (Mesocrisetus auratus L.), fed diets (crude protein; $\mathrm{PB}=13.5$ $19.6 \%$ ) with red worm (HL) (Eisenia spp.) meal and two conventional feeds (Conejarina ${ }^{\circledR}$; $\mathrm{PB}=$ $11.4 \%$ and Ratarina $\AA ; P B=21.1 \%$ ). The PT, Alb, Glb and Ct concentration oscillated among 5-6, 2$3,2-3 \mathrm{~g} / \mathrm{dL}$ y $0,3-0,4 \mathrm{mg} / \mathrm{dL}$, respectively, no significant differences among the treatments in the assay were observed $(p>0.05)$. In the assay the $U$ and $A U$ content fluctuated between 23-101 y 3-9 mg/dL, respectively; according to the PB level, $\mathrm{HL}$ inclusion and measurement time. The transaminase levels were high and variables during the second week of assay, according to the proteic level diets (TGO: 101-699 y TGP: 54-890 UI/ L). The seric PT, Glb, Alb and Ct content were independent of $\mathrm{PB}$ diets and $\mathrm{HL}$ proportion, while the $U\left(R^{2}=0.509^{*}\right), \operatorname{TGO}\left(R^{2}=0.661^{* \star}\right)$ and $\operatorname{TGP}\left(R^{2}=\right.$ $0.524^{*}$ ) level were related with proteic level of diets, and the $U\left(R^{2}=0.502^{*}\right)$ and $A U\left(R^{2}=0.531^{*}\right)$ 
contents with the $\mathrm{HL}$ inclusion. The $\mathrm{HL}$ addition in 24,27 and $30 \%$ for making formulated proteic diets to $M$. auratus cause significant variation in the normal values of $U, A U$ and transaminases.

\section{INTRODUCCIÓN}

La evaluación de alimentos para consumo animal constituye un tópico de obligada atención para los profesionales que incursionan en las ciencias agrícolas en toda Suramérica, donde en la mayoría de los casos las principales limitantes de los sistemas de producción animal se asocian a factores relacionados con la nutrición durante los ciclos de producción y a la falta de rentabilidad económica a causa de los elevados costos de los alimentos concentrados disponibles en el mercado (Cova y García, 2006; Cova et al., 2009).

En este sentido, en las últimas décadas se han caracterizado numerosas fuentes alimenticias no convencionales con el objetivo de utilizarlas como material suplementario tanto para rumiantes como para no rumiantes (Morón-Fuenmayor et al., 2008; Savón et al., 2008). Sin embargo, los ensayos de respuesta animal en términos de ganancia de peso, selectividad y mediciones de parámetros fisiológicos y metabólicos asociado a dietas experimentales son escasos, comparado con los estudios sobre caracterización químico-nutricional integral de fuentes alimenticias (García et al., 2012).

Por otra parte, hay consenso sobre la calidad de la harina deshidratada de lombriz roja (Eisenia spp.) (HL) como fuente suplementaria de proteínas, como ha sido demostrado en varias investigaciones (Vielma et al., 2003). Sin embargo, se informan potenciales problemas de toxicidad cuando es suministrada en proporciones elevadas en las dietas (Satchell, 1983).

Aún cuando se han realizado estudios en los cuales se han incorporado HL a la dieta de diversas especies, no existe mucha información sobre la influencia de dicha fuente en el perfil bioquímico sérico de animales de laboratorio, ni tampoco en condiciones comerciales (Cova y García, 2006). En este sentido, la utilización del hámster dorado (Mesocricetus auratus L.) como modelo metabólico constituye una alternativa para evaluar alimentos experimentales, por responder rápidamente a variaciones químicas en la dieta y presentar una fisiología digestiva similar a un gran número de especies de interés comercial (De Vries, 1996).

Por tales motivos, el objetivo de esta investigación fue cuantificar los niveles de metabolitos nitrogenados séricos y otros compuestos relacionados en $M$. auratus, cuando es alimentado con dietas a base de harina de Eisenia spp. y fuentes convencionales.

\section{MATERIAL Y MÉTODOS}

El estudio se desarrolló en el Vivario del Núcleo Universitario Rafael Rangel(NURR), que pertenece a la Universidad de Los Andes (ULA), en el Sector Carmona de la ciudad de Trujillo, estado Trujillo, Venezuela y se encuentra localizado a $800 \mathrm{msnm}$, temperatura media anual de $25,2^{\circ} \mathrm{C}$ y $69 \%$ de humedad relativa.

\section{CONDICIONESDEEXPERIMENTACIÓN}

Se utilizaron 76 hámsters hembras con un peso vivo promedio de $81 \pm 8,5$ g provenientes del Bioterio de la Facultad de Ciencias de la ULA, estado Mérida, Venezuela; los cuales fueron divididos en 12 grupos de seis animales y distribuidos, cada grupo, de forma aleatoria en jaulas de acero inoxidable (45,5 $\mathrm{cm}$ de largo; $40,0 \mathrm{~cm}$ de ancho y $22 \mathrm{~cm}$ de altura), con bandejas metálicas intercambiables en la parte inferior. Durante todas las etapas del ensayo los animales fueron sometidos a ciclos de luz-oscuridad de 12 horas, suministro de agua a voluntad y oferta diaria de alimento constante (NRC, 1995). Los cuatro animales que no formaron parte de los 12 grupos señalados para el desarrollo del experimento fueron utiliza- 


\section{METABOLITOS NITROGENADOS SÉRICOS EN MESOCRICETUS AURATUS}

dos en la realización del muestreo sanguíneo inicial.

Para la adaptación a las condiciones de experimentación los animales fueron confinados una semana antes, en dicho periodo se les suministró diariamente a cada animal el equivalente a $20 \mathrm{~g}$ de una dieta peletizada (proteína bruta (PB): 11,4\%; grasas: 1,2\%; fibra detergente neutro (FDN): 44,0\%; extracto libre de nitrógeno: $64,4 \%$ y cenizas: $8,6 \%$ ) y agua ad libitum. Posteriormente al periodo pre-experimental, todos los días a las 7:00 am, y por 21 días, se suministraron las dietas en los comederos de cada jaula, después de la respectiva dosificación diaria; retirando el remanente del día anterior y las excretas producidas. Los ingredientes, formulación de dietas y tratamientos y composición química se muestran en la tabla I.

RESTRUCTURACIÓN DEL ALIMENTO Y OBTENCIÓN DELOS PELLETS

Considerando que la Ratarina ${ }^{\circledR}$ y la
Conejarina ${ }^{\circledR}$ presentan tamaños de pellet diferentes, estos alimentos fueron inicialmente re-estructurados con fines comparativos, para evitar así la influencia del tamaño del pellet en el consumo. Inicialmente los alimentos se molieron usando un molino tipo corona (14/249, marca Royal Triumph), de igual forma que para el resto de los componentes de las dietas que se utilizaron para la confección de las raciones formuladas.

Las proporciones de cada ingrediente se homogenizaron, de forma secuenciada, empleando un homogenizador eléctrico (Equifarm, marca Ultraturrax), y posteriormente se usó una peletizadora de 8 cuchillas de corte con variador electrónico de velocidad y motor de $3 \mathrm{HP}$ para la confección de los pellets. Los pellets formados fueron secados en estufa con ventilación forzada (YRH 02-3, marca Kaltein) durante 48 horas y después de enfriarse a temperatura ambiente $\left(25^{\circ} \mathrm{C}\right)$, se dosificaron las cantidades a ofertar

Tabla I. Composición porcentual y química de los ingredientes utilizados en el periodo experimental. (Percentual and chemical composition of ingredients utilized during the experimental period).

\begin{tabular}{lcccccc}
\hline Ingrediente (\%) & $\mathrm{D}_{0}$ & $\mathrm{D}_{1}$ & $\mathrm{D}_{2}$ & $\mathrm{D}_{3}$ & $\mathrm{D}_{4}$ & $\mathrm{D}_{5}$ \\
\hline Conejarina & 100 & - & - & - & - & - \\
Harina de lombriz & - & 21,00 & 24,00 & 27,00 & 30,00 & - \\
Pasto estrella & - & 24,90 & 25,0 & 23,00 & 22,62 & - \\
Gelatina & - & 38,50 & 34,48 & 26,68 & 20,58 & - \\
Carbonato de calcio & - & 0,10 & 0,12 & 0,02 & - & - \\
Fosfato monocálcico & - & 0,50 & 0,40 & 0,40 & 0,40 & - \\
Maíz (granos) & - & 10,00 & 11,00 & 17,90 & 22,00 & - \\
Melaza & - & 5,00 & 5,00 & 5,00 & 5,00 & - \\
Ratarina® & - & - & - & - & - & 100 \\
& & & & & & \\
Composición química & 114,2 & 135,0 & 154,0 & 176,0 & 196,0 & 210,9 \\
PB $(\mathrm{g} / \mathrm{kg})$ & 1254 & 1359 & 1465 & 1746 & 1944 & 2003 \\
EM (kcal/kgMS) & 440,6 & 450,8 & 437,2 & 412,4 & 400,2 & 420,3 \\
FDN $(\mathrm{g} / \mathrm{kg})$ & 2,6 & 3,0 & 3,0 & 3,0 & 3,1 & 3,1 \\
Ca $(\mathrm{g} / \mathrm{kg})$ & 1,8 & 2,0 & 1,9 & 2,0 & 2,1 & 2,0 \\
P $(\mathrm{g} / \mathrm{kg})$ & & & & & \\
\hline
\end{tabular}

PB: proteína bruta; EM: energía metabolizable; MS: materia seca; FDN: fibra detergente neutro; relación $E M: P B=1: 10$ (aproximada). 
en los comederos según el tratamiento.

Las características físicas de los pellets de cada dieta fueron las siguientes: peso $=$ $1 \pm 0,2 \mathrm{~g} /$ pellet; $\operatorname{largo}=2 \pm 0,5 \mathrm{~cm} ;$ ancho $=1 \pm 0,2$ $\mathrm{cm}$. Parámetros de dureza: absorción de agua a los 15 minutos $=66 \pm 0,2 \%(\mathrm{p} / \mathrm{p})$; distancia de fracturabilidad $=0,4 \pm 0,02 \mathrm{~cm}$ y tiempo de fracturabilidad $=0,08 \pm 0,002 \mathrm{~s}$.

\section{MANEJO DE LOS ANIMALES Y PARTICULARI- DADES DEL MUESTREO}

Todo el manejo, cuidado y mantenimiento de los hamsters se realizó siguiendo las normas vigentes para el cuidado de animales de laboratorio (Home Office, 1989).

Al inicio del ensayo y al final de cada semana (día 7, 14 y 21), en ayuno, cuatro animales por tratamiento ( 2 de cada jaula) fueron primeramente anestesiados con éter etílico (BDH, Reino Unido) para realizar la extracción de sangre.

\section{TOMADE MUESTRA Y MEDICIONES SÉRICAS}

La muestra de sangre se obtuvo por punción cardiaca, la cual se extrajo con una jeringa de plástico de $5 \mathrm{~mL}$ con aguja 21 y se recibió en tubo plástico cónico Corning (marca Corning, NY, USA) que contenía EDTA-Na $a_{4}$ al $1 \%$. Se centrifugó durante 20 minutos a $3000 \mathrm{rpm}$ en una centrífuga DINAC, Becton Dickinson (marca Sparks, DM, USA). El plasma se colectó en tubo de plástico que permaneció a $4^{\circ} \mathrm{C}$ durante todo el procesamiento y periodo previo a los análisis.

En el plasma se determinó, por métodos colorimétricos de punto final, la concentración de proteínas séricas totales (PT), albúminas (Alb), globulinas (Glb), ácido úrico $(\mathrm{AU})$, creatinina $(\mathrm{Ct})$ y urea sérica $(\mathrm{U})$; y por método enzimático los niveles de transaminasas (glutámico-oxalacética (TGO) y glutámico-pirúvica (TGP)). Todas las determinaciones analíticas se realizaron utilizando el equipo Express Plus (marca Bayer, Health Care, Australia) de análisis multi-paramédico, perteneciente al Laboratorio de Bioanálisis del Instituto Venezola- no del Seguro Social, en la ciudad de Trujillo, estado Trujillo, Venezuela.

\section{DISEÑO EXPERIMENTAL Y ANÁLISIS ESTA- DÍSTICO}

Se empleó un diseño totalmente aleatorizado para un factor (dieta: $\mathrm{D}_{0}, \mathrm{D}_{1}, \mathrm{D}_{2}, \mathrm{D}_{3}, \mathrm{D}_{4}$ $\mathrm{y}_{5}$ ). La jaula se consideró la unidad experimental y el animal constituyó la unidad de observación. Para el procesamiento de la información se utilizó el paquete estadístico SPSS versión 10.0 para Windows ${ }^{\circledR}$ (Visauta, 1998). Para comparar los tratamientos en función de las variables medidas se empleó la prueba de comparación de SNK (StudentNewman-Keuls) para un $5 \%$ de probabilidad. Para la realización de las correlaciones lineales se empleó el mismo paquete estadístico utilizando el coeficiente de correlación de Pearson.

\section{RESULTADOS}

La totalidad de los hámsters alimentados con $\mathrm{D}_{4}$ después de la segunda extracción de sangre (día 7) fueron retirados del ensayo y sacrificados, previa anestesia con éter etílico, por exhibir signos de intoxicación, condición corporal desfavorable e intranquilidad excesiva.

Los valores medios y las respectivas desviaciones estándar de cada variable medida fueron: PT: $5 \pm 0,7 \mathrm{~g} / \mathrm{dL}$; Alb: $2 \pm 0,8 \mathrm{~g} /$ $\mathrm{dL}$; Glb: $3 \pm 0,7 \mathrm{~g} / \mathrm{dL} ; \mathrm{U}: 60 \pm 20 \mathrm{mg} / \mathrm{dL} ; \mathrm{AU}$ : $5 \pm 0,2 \mathrm{mg} / \mathrm{dL} ; \mathrm{Ct}: 0,4 \pm 0,2 \mathrm{mg} / \mathrm{dL}$; TGP: $275 \pm 136 U I / L$ y TGO: $281 \pm 188 \mathrm{UI} / 1$.

Con relación al contenido de $\mathrm{PT}$, no se observaron diferencias significativas entre dietas en ninguno de los muestreos realizados $(p>0,05)$. Durante el ensayo los valores fluctuaron entre 5-6 g/dL (tabla II).

Para la concentración de Alb y Glb no se observaron diferencias significativas entre las dietas $(\mathrm{p}>0,05)$ y los valores fluctuaron entre $2-3 / d L$.

La concentración sérica de $\mathrm{Ct}$ osciló entre 0,2 y $0,4 \mathrm{mg} / \mathrm{dL}$. No se observó un efecto marcado de la dieta en la concentra- 
Tabla II. Efecto de la dieta (D) en el contenido sérico de proteínas totales, albúmina, globulinas y creatinina en M. auratus. (Effect of diet in the seric content of total protein, albumin, globulins and creatinine in $M$. auratus).

\begin{tabular}{ccccc}
\hline & $\begin{array}{c}\mathrm{PT} \\
(\mathrm{g} / \mathrm{dL})\end{array}$ & $\begin{array}{c}\mathrm{Alb} \\
(\mathrm{g} / \mathrm{dL})\end{array}$ & $\begin{array}{c}\mathrm{Glb} \\
(\mathrm{g} / \mathrm{dL})\end{array}$ & $\begin{array}{c}\mathrm{Ct} \\
(\mathrm{mg} / \mathrm{dL})\end{array}$ \\
\hline Inicio & $5 \pm 3,1$ & $2 \pm 0,4$ & $3 \pm 0,8$ & $0,3 \pm 0,04$ \\
Día 7 & & & & \\
$\mathrm{D}_{0}$ & $5 \pm 2,1$ & $2 \pm 0,4$ & $3 \pm 0,7$ & $0,4 \pm 0,03$ \\
$\mathrm{D}_{1}$ & $5 \pm 2,2$ & $2 \pm 0,3$ & $3 \pm 0,6$ & $0,3 \pm 0,02$ \\
$\mathrm{D}_{2}$ & $5 \pm 2,4$ & $2 \pm 0,3$ & $2 \pm 0,5$ & $0,4 \pm 0,03$ \\
$\mathrm{D}_{3}$ & $5 \pm 2,6$ & $2 \pm 0,4$ & $3 \pm 0,6$ & $0,4 \pm 0,04$ \\
$\mathrm{D}_{5}$ & $5 \pm 2,0$ & $2 \pm 0,4$ & $3 \pm 0,7$ & $0,4 \pm 0,03$ \\
Día 14 & & & & \\
$\mathrm{D}_{0}$ & $6 \pm 2,2$ & $2 \pm 0,4$ & $3 \pm 0,7$ & $0,4 \pm 0,03$ \\
$\mathrm{D}_{1}$ & $5 \pm 2,3$ & $2 \pm 0,4$ & $3 \pm 0,8$ & $0,3 \pm 0,03$ \\
$\mathrm{D}_{2}$ & $5 \pm 2,4$ & $2 \pm 0,5$ & $3 \pm 0,8$ & $0,3 \pm 0,02$ \\
$\mathrm{D}_{3}$ & $5 \pm 2,1$ & $2 \pm 0,3$ & $3 \pm 0,8$ & $0,3 \pm 0,02$ \\
$\mathrm{D}_{5}$ & $6 \pm 2,0$ & $2 \pm 0,4$ & $3 \pm 0,7$ & $0,4 \pm 0,03$ \\
Día 21 & & & & \\
$\mathrm{D}_{0}$ & $5 \pm 2,2$ & $2 \pm 0,3$ & $3 \pm 0,7$ & $0,4 \pm 0,04$ \\
$\mathrm{D}_{1}$ & $5 \pm 2,3$ & $2 \pm 0,4$ & $3 \pm 0,6$ & $0,3 \pm 0,03$ \\
$\mathrm{D}_{2}$ & $5 \pm 2,5$ & $2 \pm 0,3$ & $3 \pm 0,7$ & $0,4 \pm 0,03$ \\
$\mathrm{D}_{3}$ & $5 \pm 2,4$ & $2 \pm 0,4$ & $3 \pm 0,7$ & $0,4 \pm 0,02$ \\
$\mathrm{D}_{5}$ & $5 \pm 2,2$ & $3 \pm 0,4$ & $3 \pm 0,7$ & $0,4 \pm 0,03$ \\
\hline
\end{tabular}

Media desviación estándar sin diferencias significativas entre dietas $(p>0,05)$.

PT: proteínas totales; Alb: albúmina; Glb: globulinas; Ct: creatinina.

ción de este metabolito ( $\mathrm{p}>0,05)$.

Por otra parte, en la tabla III se muestra el efecto de las dietas en los niveles de U, AU, TGO y TGP de los animales.

Los animales sometidos a la dieta con elevado contenido de proteínas $\left(\mathrm{D}_{5}\right)$, exhibieron mayor concentración de U plasmática, que los correspondientes al resto de los tratamientos $(\mathrm{p}<0,05)$. Los hámsters sometidos a niveles proteicos bajos e intermedios a base de $\mathrm{HL}\left(\mathrm{D}_{1}, \mathrm{D}_{2} \mathrm{y}_{3}\right)$, presentaron valores intermedios de este metabolito. Sin embargo, los animales alimentados con $\mathrm{D}_{0}$, los cuales consumieron el alimento con el menor contenido de proteínas $(11,4 \%)$ mos- traron las concentraciones más bajas de U sérica.

Con respecto a los niveles de $\mathrm{AU}$, los hámsters alimentados con $\mathrm{D}_{3}$ y $\mathrm{D}_{5}$ exhibieron mayor concentración sanguínea de $\mathrm{AU}$ $(p<0,05)$. El contenido de este compuesto en los animales sometidos al resto de los tratamientos experimentales mostraron diferencias significativas entre sí, pero con valores medios inferiores a los anteriores $(\mathrm{p}>0,05)$.

Se observó una tendencia de aumento de la concentración de TGO hasta los siete

Tabla III. Efecto de la dieta (D) en el contenido sérico de urea, ácido úrico, TGO y TGP en $\mathrm{M}$. auratus. (Interaction diet $\mathrm{x}$ measurement time in the seric content of urea, uric acid, TGO and TGP in M. auratus)

\begin{tabular}{ccccc}
\hline & $\begin{array}{c}\mathrm{U} \\
(\mathrm{mg} / \mathrm{dL})\end{array}$ & $\begin{array}{c}\mathrm{AU} \\
(\mathrm{mg} / \mathrm{dL})\end{array}$ & $\begin{array}{c}\text { TGO } \\
(\mathrm{UI} / \mathrm{L})\end{array}$ & $\begin{array}{c}\mathrm{TGP} \\
(\mathrm{UI} / \mathrm{L})\end{array}$ \\
\hline Inicio & $53 \pm 10,3$ & $3 \pm 1,1$ & $265 \pm 81$ & $214 \pm 78$ \\
Día7 & & & & \\
$\mathrm{D}_{0}$ & $43 \pm 8,3^{\mathrm{d}}$ & $4 \pm 0,3^{\mathrm{c}}$ & $139 \pm 26^{\mathrm{d}}$ & $188 \pm 32^{\mathrm{d}}$ \\
$\mathrm{D}_{1}$ & $64 \pm 10,2^{\mathrm{c}}$ & $4 \pm 0,2^{\mathrm{c}}$ & $330 \pm 95^{\mathrm{c}}$ & $430 \pm 95^{\mathrm{c}}$ \\
$\mathrm{D}_{2}$ & $71 \pm 10,1^{\mathrm{b}}$ & $6 \pm 0,7^{\mathrm{a}}$ & $383 \pm 98^{\mathrm{c}}$ & $315 \pm 73^{\mathrm{c}}$ \\
$\mathrm{D}_{3}$ & $78 \pm 9,3^{\mathrm{b}}$ & $6 \pm 0,9^{\mathrm{a}}$ & $538 \pm 54^{\mathrm{b}}$ & $622 \pm 91^{\mathrm{b}}$ \\
$\mathrm{D}_{5}$ & $79 \pm 10,4^{\mathrm{a}}$ & $6 \pm 1,1^{\mathrm{a}}$ & $634 \pm 73^{\mathrm{a}}$ & $780 \pm 103^{\mathrm{a}}$ \\
Día 14 & & & & \\
$\mathrm{D}_{0}$ & $44 \pm 7,9^{\mathrm{a}}$ & $5 \pm 0,8^{\mathrm{b}}$ & $117 \pm 19^{\mathrm{d}}$ & $139 \pm 32^{\mathrm{c}}$ \\
$\mathrm{D}_{1}$ & $71 \pm 5,8^{\mathrm{b}}$ & $5 \pm 0,3^{\mathrm{b}}$ & $208 \pm 32^{\mathrm{c}}$ & $280 \pm 45^{\mathrm{b}}$ \\
$\mathrm{D}_{2}$ & $86 \pm 8,9^{\mathrm{a}}$ & $6 \pm 0,4^{\mathrm{b}}$ & $230 \pm 46^{\mathrm{c}}$ & $291 \pm 48^{\mathrm{b}}$ \\
$\mathrm{D}_{3}$ & $83 \pm 9,8^{\mathrm{a}}$ & $9 \pm 0,4^{\mathrm{a}}$ & $317 \pm 87^{\mathrm{b}}$ & $277 \pm 54^{\mathrm{b}}$ \\
$\mathrm{D}_{5}$ & $96 \pm 10,4^{\mathrm{a}}$ & $9 \pm 0,9^{\mathrm{a}}$ & $390 \pm 80^{\mathrm{a}}$ & $473 \pm 54^{\mathrm{a}}$ \\
Día 21 & & & & \\
$\mathrm{D}_{0}$ & $23 \pm 9,5^{\mathrm{c}}$ & $3 \pm 1,2^{\mathrm{c}}$ & $101 \pm 32^{\mathrm{c}}$ & $141 \pm 41^{\mathrm{b}}$ \\
$\mathrm{D}_{1}$ & $38 \pm 5,1^{\mathrm{b}}$ & $4 \pm 0,2^{\mathrm{bc}}$ & $120 \pm 29^{\mathrm{c}}$ & $170 \pm 57^{\mathrm{a}}$ \\
$\mathrm{D}_{2}$ & $45 \pm 6,7^{\mathrm{a}}$ & $5 \pm 0,2^{\mathrm{b}}$ & $129 \pm 43^{\mathrm{c}}$ & $54 \pm 23^{\mathrm{d}}$ \\
$\mathrm{D}_{3}$ & $4 \pm 6,0^{\mathrm{a}}$ & $8 \pm 0,3^{\mathrm{a}}$ & $195 \pm 35^{\mathrm{b}}$ & $104 \pm 40 \mathrm{~b}^{\mathrm{c}}$ \\
$\mathrm{D}_{5}$ & $46 \pm 6,1^{\mathrm{a}}$ & $8 \pm 1,1^{\mathrm{a}}$ & $202 \pm 39^{\mathrm{a}}$ & $112 \pm 37^{\mathrm{c}}$ \\
$\mathrm{D}_{5}$ & & &
\end{tabular}

Media \pm desviación estándar con letras distintas entre filas, en cada momento de muestreo, indican diferencias significativas $(p<0,05)$.

$\mathrm{U}$ : urea; $\mathrm{AU}$ : ácido úrico; TGO: transaminasa glutámico oxal-acética; TGP: transaminasa glutámico-pirúvica. 
días y disminución paulatina de los niveles de esta transaminasa hasta el final del experimento con todas las dietas, exceptuando con $\mathrm{D}_{0}$ donde los niveles permanecieron estables durante el ensayo.

El contenido de TGO fue significativamente superior en los animales alimentados con $\mathrm{D}_{5}(\mathrm{p}<0,05)$, seguido por los que consumieron la dieta $\mathrm{D}_{3}$. No se observaron variaciones sustanciales entre los niveles de TGO de los hámsters sometidos a $\mathrm{D}_{1}$ y $\mathrm{D}_{2}$ en ningún momento de evaluación $(\mathrm{p}>0,05)$. Sin embargo, los animales que consumieron $\mathrm{D}_{0}$, exhibieron la menor concentración de esta enzima en la sangre durante todo el ensayo.

La tendencia del contenido sanguíneo de TGP fue similar a la observada para la TGO. En este sentido, se observaron mayores concentraciones de TGP en los animales alimentados con $\mathrm{D}_{5}(\mathrm{p}<0,05)$. Sin embargo, con la dieta menos proteica $\left(\mathrm{D}_{0}\right)$ se observó el contenido de TGP más bajo.

El nexo entre las variables medidas en la sangre; así como con los niveles proteicos de las dietas y el porcentaje de inclusión de la HL se muestran en la tabla IV.

El contenido de U y AU sérico se relacio- nó positivamente con el porcentaje de inclusión de HL. Sin embargo, la concentración de U, TGP y TGO exhibió una relación positiva con el nivel proteico de los alimentos.

Las PT presentaron un nexo positivo con el contenido de Alb; mientras que la concentración de esta última se relacionó de forma negativa con los valores de Glb. Las Alb y Glb exhibieron un nexo sustancial con el contenido de Ct, TGP y TGO; además, las Glb se relacionaron positivamente con los valores de U.

La concentración de $U$ presentó una relación fuertemente positiva con los contenidos de Ct, TGP y TGO. El contenido de Ct exhibió nexo negativo con las transaminasas; mientras que la concentración de ambas enzimas tuvo relación positiva entre sí.

\section{DISCUSIÓN}

En sentido general, los valores promedios de PT, Alb, U, AU y Ct durante todo el experimento coinciden con lo informado por numerosos autores como normales para $M$. auratus $(\mathrm{PT}: 7 \pm 0,5 \mathrm{~g} / \mathrm{dL}$; Alb: $4 \pm 0,2 \mathrm{~g} / \mathrm{dL}$, Glb: $3 \pm 0,2 \mathrm{~g} / \mathrm{dL} ; \mathrm{U}: 51 \pm 8 \mathrm{mg} / \mathrm{dL} ; \mathrm{AU}: 6 \pm 2 \mathrm{mg} /$ $\mathrm{dL} ; \mathrm{Ct}: 1 \pm 0,4 \mathrm{mg} / \mathrm{dL}$ ) (Mitruka y Rawnsley;

Tabla IV. Correlaciones entre el porcentaje de inclusión de HL, el contenido de proteína de las dietas y las variables séricas en $\mathrm{M}$. auratus. (Correlations among inclusion percentage of $\mathrm{HL}$, protein content of diets and seric variables in M. auratus).

\begin{tabular}{|c|c|c|c|c|c|c|c|c|c|}
\hline & PB & PT & Alb & Glb & U & $\mathrm{AU}$ & $\mathrm{Ct}$ & TGO & TGP \\
\hline$\% \mathrm{HL}$ & 0,042 & $-0,393$ & $-0,223$ & $-0,006$ & $0,502^{*}$ & $0,531^{*}$ & $-0,214$ & 0,231 & 0,152 \\
\hline PB & - & $-0,140$ & 0,025 & $-0,044$ & $0,509^{*}$ & 0,121 & 0,048 & $0,661^{* *}$ & $0,524^{*}$ \\
\hline PT & - & - & $0,703^{* *}$ & 0,024 & $-0,026$ & 0,073 & 0,100 & $-0,428$ & $-0,339$ \\
\hline Alb & - & - & - & $-0,606^{*}$ & $-0,307$ & 0,061 & $0,657^{* *}$ & $-0,612^{*}$ & $-0,602^{*}$ \\
\hline $\mathrm{Glb}$ & - & - & - & - & $0,558^{*}$ & 0,062 & $-0,916^{\star *}$ & $0,509^{*}$ & $0,592^{*}$ \\
\hline$U$ & - & - & - & - & - & 0,483 & $-0,611^{*}$ & $0,728^{\star *}$ & $0,695^{\star *}$ \\
\hline $\mathrm{AU}$ & - & - & - & - & - & - & 0,000 & 0,064 & 0,012 \\
\hline $\mathrm{Ct}$ & - & - & - & - & - & - & - & $-0,576^{*}$ & $-0,648^{*}$ \\
\hline TGO & - & - & - & - & - & - & - & - & $0,962^{* *}$ \\
\hline
\end{tabular}

HL: harina de lombriz; PB: proteína bruta; PT: proteína total; Alb: albúminas; Glb: globulinas; U: urea; AU: ácido úrico; Ct: creatinina; TGO: transaminasa glutámico oxal-acética; TGP: transaminasa glutámicopirúvica.

Archivos de zootecnia vol. 61, núm. 234, p. 168. 
1981; Lima et al., 1985; Ragan, 1989; CCAC, 2009). No obstante, los valores de transaminasas se consideran muy elevados comparados con los contenidos estándares informados para hámsters adultos (TGP: 35 \pm 2 UI/L; TGO: 79 \pm 32 UI/L) (CCAC, 2009). Estos valores de transferasas fueron altos con todas las dietas, inclusive al inicio del experimento y con los alimentos que no contuvieron HL, lo cual describe, independiente del efecto de los tratamientos en las variaciones del contenido de estas enzimas, la influencia de factores intrínsecos de los animales utilizados en el ensayo.

Valores anormales, en este sentido, también han sido informados por De Jesús y Quintero (2008) en la evaluación de algunos parámetros séricos en hámsters provenientes del mismo Bioterio de la ULA, del estado Mérida, Venezuela. Estas irregularidades en algunos de los indicadores sanguíneos de las camadas se atribuyeron a factores genéticos en los animales, a particularidades del sistema de producción de estos roedores en el Bioterio y a los progenitores específicos utilizados en la obtención de cada corte.

Por otra parte, la elevada desviación estándar de la media, sobretodo en el caso de los niveles de U, AU, TGP y TGO; describen fluctuaciones apreciables en relación a la composición químico-nutricional de las diferentes dietas y los muestreos secuenciales realizados durante el ensayo. Al respecto, en numerosas investigaciones realizadas con diferentes especies de animales se ha informado una relación importante entre los valores de proteína dietética, su consumo y la concentración sérica de U; así como un nexo inverso entre el contenido sanguíneo de este metabolitos y la calidad de la proteína suministrada (Beames y Eggum, 1981; Burn et al., 1982; Mariner, 1982; Thacker et al., 1982; Myung-Hee et al., 1987; Roseler et al., 1993).

La poca variación en el contenido de PT entre dietas; así como de sus fracciones, sugiere que en este tipo de roedor, la sínte- sis por parte del hígado de proteínas séricas a partir de fuentes alimenticias con diferentes niveles proteicos $(11,4-21,1 \%)$, es relativamente constante y no se encuentra influenciada directamente por la proporción de los componentes proteicos mayoritarios, ni por la presencia de otros compuestos nitrogenados presentes en la sangre. Este comportamiento se ha relacionado con la disponibilidad de algunos aminoácidos en las proteínas dietéticas, ya que en su ausencia ocurre un punto de ruptura y la concentración, tanto de proteínas séricas, como de otros compuestos relacionados con el ciclo de la U, disminuyen abruptamente; independientemente del valor neto de proteína de la ración (Kirk y Walter, 1976a,b; Kumar et al., 1980; Myung-Hee et al., 1987; Kesteloot and Joossens, 1993). Quizás por ese motivo, no se observaron fluctuaciones importantes en las concentraciones de proteínas séricas entre dietas, si se considera que los requerimientos proteicos, en términos de aminoácidos, se encontraron cubiertos durante todo el ensayo en función de la oferta y el consumo (García et al., 2012).

Por otra parte, la Alb (seroalbúmina) es la principal proteína de la sangre y a su vez la más abundante en muchos animales. Los niveles de estas proteínas son muy variables, de manera que sus concentraciones en el plasma podrían no reflejar el estado nutricional; pero sí del grado de severidad de trastornos de órganos relacionados con la síntesis y excreción (De Vries, 1996). Considerando estas razones, la estabilidad en los valores de estas proteínas no indicaron efectos adversos de las dietas suministradas acorde al gradiente proteico (Toledo et al., 2005).

Por otra parte, las Glb son un grupo de proteínas insolubles en agua que se encuentran en todos los animales. Entre las Glb más importantes destacan las seroglobulinas (de la sangre), el fibrinógeno y los anticuerpos ( $\alpha$-globulinas). Éstas se pueden dividir en varios grupos (globulinas $\alpha$ - 
$1, \alpha-2, \beta y \gamma)$, la mayoría se sintetizan también en el hígado y una menor parte en algunas células plasmáticas (Dietschy et al., 1993). En muchos casos sus niveles bajan dentro del rango considerado como normal, a expensa del aumento de las concentraciones de Alb, para que se mantengan los niveles de proteínas séricas constantes (relación: Alb/Glb), sin que se manifieste ninguna patología de origen inmune; lo que parece ser la razón por la cual en todos los tratamientos se observó una estabilidad en los valores de la concentración de estas proteínas, aún dentro de los contenidos estándares para estos roedores, sin diferencias entre tratamientos.

En otro orden, la influencia evidente de los niveles proteicos dietéticos en los valores de U se explica por el hecho de que este metabolito es el compuesto final de desecho del catabolismo de las proteínas (Gross et al., 1982). Al respecto, el nitrógeno del organismo procede siempre de las proteínas que sufren el proceso de desaminación y transaminación, el cual por medio del metabolismo se transforma en amoníaco $\left(\mathrm{NH}_{3}\right)$, la $\mathrm{U}$, el AU, la creatina, la Ct, el ácido hipúrico y los cuerpos sulfurados (De Vries, 1996).

En los mamíferos, la U es, en general, responsable de la excreción del $80 \%$ del nitrógeno no proteico que se excreta por la orina (Bassilyn et al., 1982); por lo que el dosaje de la U sérica normalmente se usa para evaluar la función renal y hepática. Considerando los resultados obtenidos, los valores de $\mathrm{U}$ en los animales que consumieron $\mathrm{D}_{2}, \mathrm{D}_{3} \mathrm{y} \mathrm{D}_{4}$, en la segunda y tercera medición, sugieren variaciones condicionadas por el funcionamiento deficiente del hígado y/o los riñones, evidencia encontrada también en estudios anteriores donde se evaluó la morfometría de órganos en estos hámsters (García et al., 2012).

Adicionalmente, el aumento de la cantidad de U en sangre es debido a la reducción de la eliminación renal, y también al aumento del catabolismo de proteínas, o a la combinación de esos dos factores (De Vries, 1996).
En este caso, quizás el aumento de los niveles de U se debió a estas causas, si se tienen en cuenta las evidencias encontradas con relación al aumento de peso y coloración atípica de los riñones e hígado; así como el aumento de peso, e hipertrofia hepática de los hamsters, lo cual se ha encontrado asociado a las mayores proporciones de HL en las dietas (Cova y García, 2006; García et al., 2012).

Por otra parte, la producción de U pasa por la desaminación de los aminoácidos o por la acción bacteriana en el intestino, lo cual es otro elemento a tener en cuenta ya que también se describió en ensayos anteriores anomalías en el intestino, inflamaciones y necrosis tisular en los hámsters que consumieron $\mathrm{D}_{2}, \mathrm{D}_{3}$ y $\mathrm{D}_{4}$ (García et al., 2012).

En otro orden de ideas, el ciclo de la U debe estar convenientemente regulado para poder equilibrar el balance nitrogenado del organismo. Esta regulación se puede realizar controlando las cantidades de enzimas que actúan en el ciclo. Esto quizás se relacione con las variaciones abruptas que se observaron en el contenido de TGO y TGP durante el segundo muestreo y su disminución progresiva en el tiempo hasta el final del experimento, aún cuando los niveles de $\mathrm{U}$ y AU disminuyeron significativamente sólo en la cuarta medición.

Con dietas ricas en proteínas, tales como $\mathrm{D}_{2}, \mathrm{D}_{3}, \mathrm{D}_{4} \mathrm{y} \mathrm{D}_{5}$, en el sistema digestivo se aumenta la degradación de aminoácidos, generándose más $\mathrm{NH}_{3}$, y en esta situación se produce un aumento de la síntesis de enzimas, mientras que si el alimento es de menor contenido proteico $\left(\mathrm{D}_{0}\right)$, disminuye la cantidad de orina y los valores de las enzimas que participan en el ciclo (De Vries, 1996).

También es conocido que en los estados de ayuno, donde la degradación es exclusiva de proteínas endógenas por necesidades energéticas, se incrementan los valores de enzimas del ciclo de la U (Myung-Hee et al., 1987). Quizás por este motivo en todos los tratamientos los niveles de TGP y TGO fueron más elevados que los normales, consi- 


\section{METABOLITOS NITROGENADOS SÉRICOS EN MESOCRICETUS AURATUS}

derando que la extracción de sangre siempre se realizó antes de que se ofertaran los alimentos a los animales en sus respectivas jaulas.

Por otra parte, el AU es el metabolito principal de las purinas en los mamíferos. El incremento de sus niveles séricos muestra, en muchos casos, insuficiencia renal y conlleva al aumento de la U sanguínea. Con niveles cercanos a $15 \mathrm{mg} / \mathrm{dL}$ puede producirse obstrucción, lesiones, hipogenesia renal, insuficiencia cardiaca congestiva, hipotiroidismo, hipertensión, psoriasis, y también hiperparatiroidismo. En este sentido, en las dietas con mayores proporciones de $\mathrm{HL}\left(\mathrm{D}_{3}\right.$ y $\left.\mathrm{D}_{4}\right)$ y la más proteica $\left(\mathrm{D}_{5}\right)$ se observaron valores cercanos a $10 \mathrm{mg} / \mathrm{dL}$, sobretodo en las dos últimas mediciones, lo cual sugiere posibles efectos negativos asociado al contenido de PB y la inclusión en proporciones elevadas de HL.

$\mathrm{La} C t$ es el elemento nitrogenado de la sangre menos variable, ya que los daños y perjuicios pequeños que ocurren a nivel renal no son capaces de promover sus alteraciones séricas (Muñoz et al., 2008). La Ct se forma a partir de la fosfocreatina muscular, que tiene su origen en los aminoácidos arginina y glicina, siendo, por consiguiente, un producto endógeno del catabolismo de la creatina a nivel muscular. Se elimina por los riñones en cantidades constantes a través de la filtración glomerular. En sentido general, sus valores son independientes de la ingestión de proteínas y no son afectados por el volumen urinario (Kirkpatric et al., 1975), aspectos que coinciden con los resultados obtenidos en este ensayo.

De esta manera, los estudios bioquímicos de PT, Alb, Ct, U, AU y transaminasas son buenos indicadores de los efectos perjudiciales de ingredientes dietéticos $\mathrm{u}$ otras sustancias (Burn et al., 1982; Toledo et al., 2005); considerando que en los animales alimentados con las dietas donde se incluyó HL al 24,27 y $30 \%$, en estudios previos, se observaron alteraciones en el tracto gastrointestinal; así como baja digestibilidad y pérdida de peso, atribuido a la presencia de aminas biogénicas en la HL (García et al., 2012).

No obstante, es importante verificar que factores tales como la dominancia, el linaje, la edad, el estado fisiológico y sexo de los animales; así como las condiciones ambientales, no influyan en los valores de estos parámetros sanguíneos, ya que las variaciones en el perfil metabólico sérico se pueden atribuir a las dietas cuando otros factores que no se consideran oportunamente pueden influir. En tal sentido, es fundamental, por lo tanto, considerar los valores encontrados al inicio del ensayo y en el grupo alimentado con $\mathrm{D}_{0}$, como valores de partida para realizar comparaciones en función de las dietas, ya que los niveles hematológicos y de química sanguínea de hámsters varían comúnmente respecto a valores internacionales en función de las condiciones de experimentación (De Jesús y Quintero, 2008).

Por otra parte, los fluctuantes valores de transaminasas también sugieren alteraciones hepáticas fundamentalmente en la segunda medición. Al respecto, las transaminasas (aminotransferasas) son un conjunto de enzimas, que transfieren grupos amino desde un metabolito a otro, generalmente aminoácidos. Estas enzimas son inducibles, porque su actividad puede aumentarse por la acción de diversas hormonas y su reacción es libremente reversible (De Vries, 1996).

En tal sentido, los niveles de transaminasas en sangre se utilizan, como indicador, para detectar posibles patologías en las funciones del hígado. Las enfermedades hepáticas: hepatitis viral, cirrosis, hígado graso, el consumo de tóxicos o compuestos pro-tóxicos y la presencia de quistes o citoanomalías en el hígado pueden provocar un aumento notable de las transaminasas en sangre (Rees y Watson, 2000). Enfermedades hepáticas provocan un aumento notable de la TGP en el plasma sanguíneo, mientras que si ocurre degeneración tisular se produce un incremento más marcado de la TGO. En este experimento el incremento significativo de la TGO y TGP en el segundo 
muestreo sugiere alteraciones sustanciales en el hígado y posiblemente en otros órganos, este efecto pudo ser reversible ya que se observó disminución progresiva de las concentraciones de enzimas en la tercera y la cuarta medición, quizás por restablecerse, a mediano plazo, el equilibro enzimático mediante mecanismos homeostáticos (Toledo et al., 2005).

Con relación al nexo entre las variables séricas, el contenido proteico de las dietas y el porcentaje de inclusión de HL; la relación entre los niveles de U, TGP y TGO con el nivel de PB dietética quizás evidencia un efecto catabólico aumentado en los animales durante las dos primeras semanas del ensayo, aspecto que ha sido señalado como la principal causa de valores anómalos de enzimas del grupo de las transferasas y de metabolitos nitrogenados relacionados con el ciclo de la U (Ragan, 1989; Roseler et al., 1993). En cambio, el nexo positivo entre la concentración de AU y la inclusión de la HL se explica por el hecho de que este ácido orgánico es el compuesto principal de la degradación de las bases nitrogenadas presentes en las harinas de carne (Rees y Watson, 2000; Cova y García, 2006).

La relación negativa entre el contenido de TGP y TGO con la Ct describe posiblemente la recuperación hepática que tuvo lugar a mediano plazo, aunado a un mayor desarrollo muscular de los animales. Resultados que apoyan lo expresado por Cova y García (2006) en estudios similares.

En este sentido, se ha informado que durante la recuperación del organismo a partir de traumas metabólicos alimentarios o patológicos, los niveles de los principales compuestos involucrados en la anomalía, así como las enzimas relacionadas con la síntesis y degradación de estos metabolitos, se estabilizan a mediano y largo plazo como

\section{BIBLIOGRAFÍA}

Bassilyn, S., Michaelk, G. and Said, A.K. 1982. Blood urea content for evaluating dietary protein consecuencia del restablecimiento de los procesos fisiológicos (Rees y Watson, 2000).

\section{CONCLUSIONES}

En función de las variables medidas, la alimentación de $M$. auratus con una dieta baja en PB $\left(D_{0}\right)$ produjo valores bajos en el perfil nitrogenado sérico de los animales. Sin embargo, con la inclusión de HL al 24, 27 y $30 \%$ se observaron valores superiores a los establecidos como normales para la especie, y con $\mathrm{D}_{1}$ los niveles sanguíneos se mantuvieron dentro del rango informado por la literatura para este roedor.

Durante la primera semana de suministro de las dietas experimentales el perfil metabólico, en término de los compuestos nitrogenados y las aminotransferasas, reflejaron alteraciones sustanciales, lo cual sugiere variaciones causadas por las dietas que exhibieron elevada proporción de HL; así como por la más proteica $\left(D_{5}\right)$.

\section{AGRADECIMIENTOS}

Al Consejo de Desarrollo Científico, Humanístico, Tecnológico y de las Artes de la Universidad de los Andes (CDCHTAULA) por el financiamiento otorgado para la realización de esta investigación (CDCHTNURR-C-355-04-03-A). A la Estación Experimental y de Producción Agrícola Rafael Rangel por el apoyo recibido para la obtención de la harina utilizada en el ensayo. Al laboratorio del Centro Nacional de Investigaciones Agropecuarias (INIA-CENIAPMaracay, Venezuela) por su colaboración en algunas de las determinaciones analíticas. Al Vivario del Núcleo Universitario Rafael Rangel del estado Trujillo, Venezuela, en la persona del Sr. Javier Moncayo, por su colaboración en el mantenimiento de los animales durante el periodo experimental.

quality. Nahrung, 26: 759-764.

Beames, R.M. and Eggum, B.O. 1981. The effect 


\section{METABOLITOS NITROGENADOS SÉRICOS EN MESOCRICETUS AURATUS}

of type and level of protein, fiber and starch on nitrogen excretion patterns in rats. Br J Nutr, 46: 301-313.

Burn, S.R., Lefaivre, M.H. and Milner, I.A. 1982. Effects of dietary protein quantity and quality on the growth of dogs and rats. J Nutr, 112: 18431853.

CCAC. 2009. Canadian Council on Animal Care. Valores de referencia de bioquímica clínica. Editorial CCAC. Ontario. Canadá. 5 pp.

Cova, L.J. y García, D.E. 2006. Evaluación de parámetros nutricionales utilizando harina de lombriz (Eisenia spp.) en la alimentación de hámster (Mesocricetus auratus L.). Jornada Técnica, Estación Experimental y de Producción Agrícola Rafael Rangel (EEPARR)-Instituto Nacional de Investigaciones Agrícolas (INIA). Trujillo. Venezuela. $12 \mathrm{pp}$

Cova, L.J., García, D.E., Scorza D., J.V., Medina, M.G., Clavero, T., Perea, F. y González, D. 2009. Efecto de la estrategia de conservación en la calidad nutritiva de la harina de la lombriz roja (Eisenia spp.) a mediano plazo. Rev Fac Agron LUZ, 26: 107-128.

De Jesús, R. y Quintero, Z. 2008. Comparación del crecimiento, parámetros reproductivos y de hematología y glicemia en hámsters (Mesocricetus auratus) alojados a diferentes niveles de intensidad de luz. Zootec Trop, 26: 19-26.

De Vries, J. 1996. Food safety and toxicity. De Vries. J. (Ed.). London, UK. 338 pp.

Dietschy, J.M., Turley, S.D. and Spady, D.K. 1993. Role of liver in the maintenance of cholesterol and low density lipoprotein homeostasis in different animal species, including humans. Lipid Res, 34: 1637-1659.

García, D.E., Cova, L.J., Castro, A.R., Medina, M.G. y Palma, J.R. 2009. Efecto del sustrato alimenticio en la composición química y el valor nutritivo de la harina de la lombriz roja (Eisenia spp.). Rev Cient LUZ, 29: 55-62.

García, D.E., Cova, L.J., Scorza D., J.V., González, M.E., Pizzani, P., Medina, M.G., Perea, F. y González, D. 2012. Efecto de dietas proteicas a base de harina de lombriz roja (Eisenia spp.) en el comportamiento del hámster dorado (Mesocricetus auratus L.). I. Dinámica de peso y digestibilidad. Rev Fac Agron LUZ, 29: 249-273.

Gross, R., Schoenebergehr, H.R. and Malaga, I. 1982. A short term urea assay (STUA) to evaluate the protein quality of food. Nutr Rep Int, 26: 629-634.

Home Office. 1989. Code of practice for the housing and care of animals used in scientific procedure. $2^{\text {nd }}$ ed. HM\&O. Londres. 200 pp.

Kesteloot, H.E. and Joossens, J.V. 1993. Relationship between dietary protein intake and serum urea, uric acid and creatinine, and 24hour urinary creatinine excretion: the BIRNH study. J Amer Coll Nutr, 12: 42-46.

Kirk, R.D. and Walker, D.M. 1976a. Plasma urea nitrogen as an indicator of protein quality. I. Factors affecting the concentration of urea in the blood of the pre-ruminant lamb. Aust J Agric Res, 27: 109-116.

Kirk, R.D. and Walker, D.M. 1976b. Plasma urea nitrogen as an indicator of protein quality. II. Relationships between plasma urea nitrogen, various urinary nitrogen constituents, and protein quality. Aust J Agric Res, 27: 117-127.

Kirkpatric, K.L., Buckland, D.E., Abler, W.A., Scanlon, P.F., Whelan, J.B. and Burkhart, H.E. 1975. Energy and protein influences on blood urea nitrogen of white-tailed deer fawns. $J$ Wildlife Manage, 39: 692-698.

Kumar, S., Mehra, U.R. and Singh, U.B. 1980. Effect of dietary protein on plasma protein, plasma protein fractions and plasma urea in ruminants. Indian J Anim Sci, 50: 146-150.

Lima, A.O., Soares, B.J., Grego, J.B., Galizzi, J. e Cançado, J.R. 1985. Método de laboratório aplicado a clinica. Técnica e Interpretação. $6^{\mathrm{a}}$ ed. Koogan. Rio de Janeiro. pp. 543.

Mariner, S. 1982. Studies on urea in serum and fertility in cows. Nutr Abstr Rev, 52: 220-221.

Mitruka, B.M. and Rawnsley, H.M. 1981. Clinical biochemical and hematological reference values in normal experimental animals and normal humans. $2^{\text {nd }}$ Ed. Masson Publishing. New York. Morón-Fuenmayor, O.E., Diaz, D., Pietrosemoli, S., Barrera, R., Gallardo, N., Peña, J. y Leal, M. 2008. Efecto de la inclusión de harina de lombriz sobre el rendimiento en canal, en cortes y calidad físico-química de la carne de codorniz (Coturnix coturnix japonica). Rev Fac Agron LUZ, 25: 674-685.

Muñoz, V., García, A.C., Herrera, J.G., Álvarez, A.G., Estrada, S.G. y Meza, M. 2008. Efecto del extracto de Yucca schidigera en el perfil bioquímico y hemático de cerdos en crecimiento 


\section{GARCÍA, COVA, BRICEÑO, SCORZA, MONTILLA, MEDINA, PEREA Y GONZÁLEZ}

y engorde. Rev Cient LUZ, 18: 51-58.

Myung-Hee, K., Morris, J.G. and Quinton, R.R. 1987. Effect of concentration of some dietary amino acids and protein on plasma urea nitrogen concentration in growing kittens. J Nutr, 117: 1689-1696.

NRC. 1995. Nutrients requirements of laboratory animal. Hamster. Fourth revised edition. National Academy Press. London, UK. 138 pp.

Ragan, HA. 1989. Markers of renal function and injury. In: Loebe, WF, Quimbly FW. (Eds). The clinical chemistry of laboratory animals. Pergamon. New York. pp. 321-344.

Rees, N. and Watson, D. 2000. International standards for food safety. Aspen Publishers, Inc. Aspen, Colorado. USA. 256 pp.

Roseler, D.K., Ferguson, J.D., Sniffen, C.J. and Herrema, J. 1993. Dietary protein degradability effects on plasma and milk urea nitrogen and milk nonprotein nitrogen in Holstein cows. $J$ Dairy Sci, 76: 525-534.

Satchell, J.E. 1983. Earthworm ecology from Darwing to vermiculture. Chapman \& Hall. Nueva York. EEUU. 485 pp.

Savón, L., Mora, L.M., Dihigo, L.E., Rodríguez, V.,
Rodríguez, Y., Scull, I., Hernández, Y. y Ruíz, T.E. 2008. Efecto de la harina de follaje de Tithonia diversifolia en la morfometría del tracto gastrointestinal de cerdos en crecimiento-ceba. Zootec Trop., 26: 387-390.

Thacker, P.A., Rowland, J.P., Milligan, L.P. and Weltzien, E. 1982. Effects of graded dietary protein levels on urea re cycling in the pig. Can J Anim Sci, 62: 1193-1197.

Toledo, T., Tanus, J., Matos, R., Machado, H., Jóia de Mello, V., Queiroga, E. y Silva, E. da. 2005. Efectos del Monascus sobre albúmina, creatinina, urea y ácido úrico en conejos. Acta Bioquím Clín Latinoam, 39: 429-434.

Vielma, J.F., Ovalles, A., León, A. y Medina, A. 2003. Valor nutritivo de la harina de lombriz (Eisenia foetida) como fuente de aminoácidos y su estimación cuantitativa mediante cromatografía en fase reversa (HPLC) y derivatización pre-columna con oftalaldehído (OPA). Ars Pharm, 44: 43-58.

Visauta, B. 1998. Análisis Estadístico con SPSS para Windows. En: Visauta B (Ed.). Estadística Multivariante. Mc-Graw-Hill-Interamericana. Madrid. España. 200 pp.

Archivos de zootecnia vol. 61, núm. 234, p. 174. 